Original Research Article

\title{
Comparison of clinical outcomes of sitagliptin+metformin combination and glimepiride in the management of uncomplicated type 2 diabetics
}

\author{
Jarinabanu Tahashildar ${ }^{1}$, Ravi Shekhar Singh ${ }^{2 *}$, Jameela Tahashildar $^{3}$
}

\begin{abstract}
${ }^{1}$ Department of Pharmacology, Dr. D.Y. Patil Medical College, Kolhapur, Maharashtra, India ${ }^{2}$ Department of Pharmacology, Dr. P.D.M. Medical College, Amravati, Maharashtra 444604, India

${ }^{3}$ Department of Pharmacology, GMC, Udaipur, Rajasthan, India
\end{abstract}

Received: 07 October 2018 Accepted: 29 October 2018

*Correspondence to: Dr. Ravi Shekhar Singh, Email: ravi.singh30@ rediffmail.com

Copyright: (C) the author(s), publisher and licensee Medip Academy. This is an openaccess article distributed under the terms of the Creative Commons Attribution NonCommercial License, which permits unrestricted noncommercial use, distribution, and reproduction in any medium, provided the original work is properly cited.

\begin{abstract}
Background: To evaluate the comparison of clinical outcomes of sitagliptin +metformin and glimepiride in uncomplicated Type-2 diabetics.

Methods: This one year (July 2016 to August 2017) prospective, open label, observational clinical cohort study was carried out on type- 2 diabetics. In this study 299 Type- 2 diabetics patients were enrolled and were randomly allocated to two groups viz Group A and Group B. Group A received sitaglitin+metformin $(50+500) \mathrm{mg} /$ day and Group B received glimepiride $1 \mathrm{mg} /$ day respectively. The follow up started after 10 days of stabilization of the patient and data recorded on 10th day was considered Zero month data and follow up continued up to Six month in each group. Comparison of FPG, PPG and HbA1c was evaluated between zero and six months within group and at six month between groups. Adverse events were recorded and summarized by treatment group.

Results: At the end of six months follow up the patients of Group A who received sitaglitin+metformin $(50+500) \mathrm{mg}$ /day had greater reduction in FPG, PPG and HbA1c (all $\mathrm{P}<0.001$ ) was recorded when compared between zero and six month within group. A significant reduction in FPG, PPG and HbA1c (all P<0.01) also recorded in Group B who received glimepiride $1 \mathrm{mg}$ /day when compared between zero and six months within group. A statically significant difference (all $\mathrm{P}<0.05$ ) was recorded at six months between group. The adverse events like hypoglycemic episodes, gastrointestinal adverse events etc were greater in Group B than Group A. Changes in weight also noted in both Groups. Weight loss in Group A and weight gain in Group B was recorded.

Conclusions: The present study suggests that a significant difference may be existing in the clinical outcome interm of glycemia control and adverse events between sitagliptin+metformin combination and glimepiride in type- 2 diabetic patients.
\end{abstract}

Keywords: Glimepiride, Glycemia control, Sitagliptin+Metformin, Type 2 DM patients

\section{INTRODUCTION}

Diabetes mellitus (DM) is a metabolic disorder characterised by defect in insulin secretion or insulin action, or both. ${ }^{1-4}$ As per WHO estimation more than 200 million people worldwide will have DM and 300 million will subsequently have the disease by $2025 .^{5-7}$ India had 32 million diabetic subjects in the year 2000 and this number would increase to 80 million by the year $2030 .{ }^{8}$ This high global burden is continuously on the rise with increasing incidence and prevalence of type 2 DM, due to increasing population, age, obesity, and physical inactivity as well as by the increasing longevity of patients with DM. Type 2 $\mathrm{DM}$ is a major risk factor for developing both 
microvascular (retinopathy, nephropathy and neuropathy) and macrovascular complications (coronary heart disease, cerebrovascular disease and peripheral vascular disease). ${ }^{9}$ Variable treatments focus on reducing hyperglycemia and improving insulin sensitivity. These modalities are attractive in theory, as they appear to target the primary defects associated with type 2 DM. However, despite the wide array of treatment options available, glycemic control declines over the time. ${ }^{10}$ Unattainable glycemic control is often a result of ongoing deterioration of beta-cell function. The primary goal of treatment is to target glycemic control by maintaining the $\mathrm{HbA} 1 \mathrm{C}$ level at 6-7\% to decrease the incidence of microvascular and macrovascular complications without predisposing patients to hypoglycemia. ${ }^{11}$ Treatment with a single antihyperglycaemic agent is often unsuccessful at achieving and/or maintaining long-term glycaemic control in patients with type 2 diabetes, so many patients require combination therapies. ${ }^{12}$ Monotherapy with metformin or a sulphonylurea is the most commonly used initial oral hypoglycaemic agent (OHA) regimen to treat patients with type 2 diabetes.

Various new drugs are introduced as monotherapy and fixed dose combinations for the treatment of type 2 diabetes. One such newly introduced class of drug is dipeptidyl peptidase-IV (DPP IV) inhibitors. Sitagliptin is a once-daily, orally active, potent and highly selective (DPP-4) inhibitor approved in many countries for the treatment of patients with type- 2 diabetes. ${ }^{13}$ It is being used as monotherapy or as an add-on to ongoing oral antidiabetic agents in patients with type $2 \mathrm{DM}$ with significant reduction in glycaemic levels within a few weeks. ${ }^{14}$ DPP IV inhibitors are being used as monotherapy or as an add-on to ongoing oral antidiabetic agents in patients with type 2 diabetes. However, there is paucity of scientific literature regarding their comparative clinical outcomes. Hence, it was found worthwhile to study and compare the clinical outcomes of combination therapy of DPP IV inhibitors i.e. sitagliptin+metformin and glimepiride in the management of uncomplicated type 2 diabetics.

\section{METHODS}

This study was carried out in the Department of Pharmacology at Geetanjali Medical College and Hospital and other Tertiary care Hospitals on type 2 diabetes mellitus patients from July 2016 to August 2017. The diagnostic criteria as presented by American diabetic association was followed and blood glucose estimation with history and clinical examination were undertaken to diagnose the diabetes mellitus. The uncomplicated Type- 2 diabetes mellitus patients of age between 18-70 years were included into the study. However, Type -I diabetes (IDDM), pregnant women, patients with impaired renal/ liver functions and the patient with history of hypersensitivity to the study drugs were excluded. Written, informed consent of all the patients and approval of
Institutional Ethics Committee (IEC) was taken before starting the study.

\section{Study design}

This study was a prospective, open label; observational clinical cohort study carried out on type 2 diabetes mellitus patients. It enrolled a total of 299 type 2 diabetes mellitus patients who were randomly allocated to two groups viz group A and group B respectively. Group A received sitagliptin+metformin $(50+500) \mathrm{mg} /$ day and group B received glimepiride $1 \mathrm{mg}$ /day respectively. A day before starting the treatment, Before Breakfast (BBF) and post prandial (PP) blood sugar was measured and treatment started. The patients were advised to come after 10 days from the start of the treatment for measurement of respective blood sugar levels and $\mathrm{HbA1c}$ again. The data so collected on the day 10 from the start of the treatment was considered as zero month. The follow up of the patients was started and the respective blood glucose levels were measured every month upto the period of six months in each patient. In addition, HbA1c was also measured and assessed after every 3 months. Blood glucose was also measured at any time if a patient experienced symptom of hypoglycaemia $(\mathrm{BG}<60 \mathrm{mg} / \mathrm{dl})$ or if requested by treating physician. Apart from glycaemia profile the complications attributable to the treatment regimens were also recorded in both the groups to assess the safety parameter.

\section{Study protocol}

As the patient turned out to be diabetic, routine investigation of fasting, random and post prandial blood glucose was done twice for confirmation. After being educated on diet, importance of treatment regimen with special emphasis on need to adhere to treatment, the patient was started with one of the two regimes. The blood glucose estimation was done by glucose oxidase test in the central laboratory of the concerned hospital by using Olympus AU 640 autoanalyser. A blood sample of $10 \mu 1$ for estimation of blood glucose was done within half an hour after the sample collection. HbA1C was also used as a comparative criteria for the assessment of glycaemia control in each patient. A proforma was developed for collecting the data required for this study. Face to face interview technique was used for interviewing the patients and / or their closest attendants. The other technique applied was that of retrospective analysis of the records. It was done with an intention to provide supplementary information on the data collected. In this study, it was contemplated to analyze the records of previous treatment with the history of diabetes mellitus to test the reliability about the duration of disease and compliance to treatment. The patient sample for this study was calculated as per the incidence of Type 2 Diabetes mellitus in the projected area and the power of study is more than $80 \%$. Unpaired t-test was employed for statistical analysis of the data. A probability value of less than $0.05 \quad(\mathrm{p}<0.05)$ was considered to be statistically significant. 


\section{RESULTS}

A total of 299 type 2 diabetes mellitus patients have completed the study. The patients were allocated to two groups Viz group A and B received sitagliptin +metformin and glimepiride respectively. Both the treatment groups had more or less similar clinical and demographic characteristics. The most common admitting outcomes assessed were glycemia control, and the incidence of adverse drug reactions. Comparison for assessment of glycemia control was done between zero and six month within group and at six month between groups.

Mean fasting blood glucose obtained a day Before Treatment (BT) has significantly reduced when compared to mean fasting blood glucose levels obtained on zero month till a period of six months within and between groups after the treatment. A significant difference exists in all the mean blood glucose levels between treatment groups.

While assessing the glycemia control a significant $(p<0.001)$ but gradual decrease was noted in all the mean blood glucose levels recorded for a period from zero month to six month when compared with the mean fasting and postprandial blood glucose levels recorded before starting the treatment in group A. A highly significant $(\mathrm{p}<0.001)$ glycemia control noted with Comparison of mean fasting and mean post prandial blood glucose levels between zero month and six month therapy within Group A.

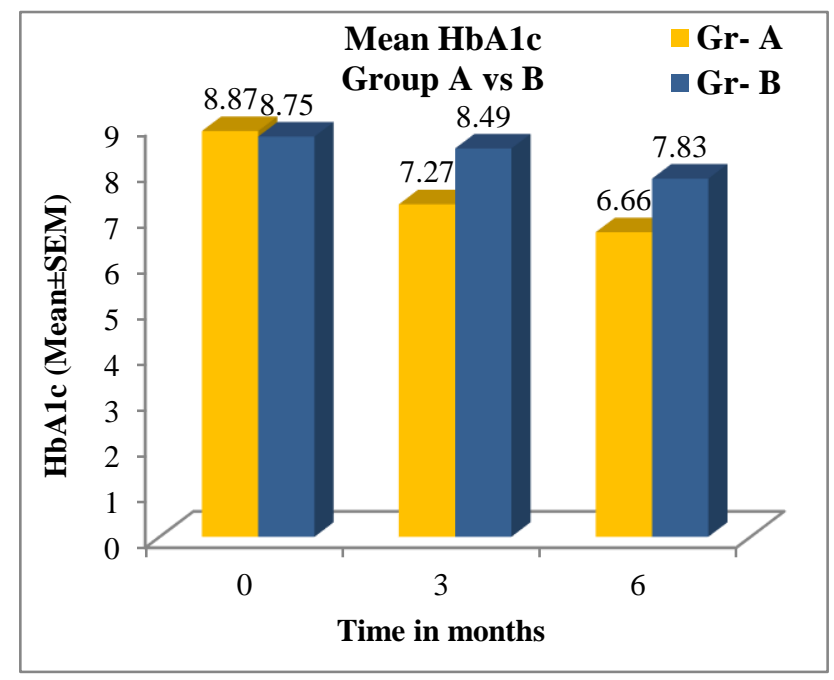

Group A= Sitagliptin+Metformin, Group B = Glimepiride

\section{Figure 1: Comparison of $\mathrm{HbA1C}$ between group $\mathbf{A}$ and $\mathbf{B}$.}

A significant decrease in all the mean blood glucose levels was recorded for a period from zero month to six month when compared with mean blood glucose levels obtained a day before starting the treatment in group B. Interstingly a highly significant $(\mathrm{p}<0.01)$ glycemia noted with comparision of mean fasting and mean ostrandial blood glucose levels between zero month and six month therapy was recorded with group B.

While assessing the overall comparative glycemia control achieved in both the treatment groups, a significant $(\mathrm{p}<0.05)$ glycemia control was noted between group A and B.

In addition, each patient was assessed for the $\mathrm{HbA} 1 \mathrm{c}$ and used as a comparative criterion for the assessment of glycaemia control in both the groups. A significant difference $(\mathrm{p}<0.001)$ existed in $\mathrm{HbA1c}$ values recorded after the three and six months period when compared to $\mathrm{HbA1c}$ value recorded on zero month period in group- A who were treated with combination of sitagliptin + metformin. A significant difference $(p<0.01)$ was also noted in $\mathrm{HbA} 1 \mathrm{c}$ values recorded before or after treatment in a group B who were treated with glimepiride alone. HbA1c assessed between groups a significant difference $(\mathrm{p}<0.05)$ was noted at six month (Figure 1).

There were 4.63 percent hypoglycaemic episodes recorded in patients who were treated with sitagliptin+metformin combination. Whereas 8.10 percent hypoglycaemic episodes were recorded in patients who were treated with glimepiride. However, this difference in hypoglycaemic episodes was statistically significant. 7.94 and 6.08 percent of the patients treated with sitagliptin+metformin combination and glimepiride respectively suffered GIT complications like nausea, vomiting and abdominal discomfort. Weight loss was recorded in 4.63 percent patients who were treated with sitagliptin + Metformin. Whereas Weight loss was not observed but weight gain was recorded in 20.94 percent patients who were treated with nation of glimepiride alone.

\section{DISCUSSION}

Diabetes mellitus is the most common endocrine disorder which is characterized by relative or absolute deficiency of insulin. The incidence of diabetes is alarming globally. India is leading the world as rank one in diabetics, thus has earned a dubious distinction of being termed as Diabetic Capital of the world. As per WHO estimation, India is going to receive 80 million diabetics in the year 2030 . Though there is no permanent treatment available for diabetes till date, however, the current treatment modalities can only control but cannot cure it. Therefore, number other new drugs are introduced as monotherapy and fixed dose combinations for the treatment of type 2 diabetes. One such newly introduced class of drug is DPP IV inhibitors.

DPP IV inhibitors are being used as monotherapy or as an add-on to ongoing oral antidiabetic agents in patients with type 2 diabetes. However, there is paucity of scientific literature regarding their comparative clinical outcome. Hence, it was found worthwhile to study and compare the clinical outcomes of Sitagliptin + metformin combination 
and with glimepiride alone in terms of glycaemia control achieved and incidence of adverse drug reactions.

A total of 299 Type 2 Diabetes Mellitus patients have completed the study. The patients were allocated to two groups Viz group A and B received combination of sitagliptin+metformin and glimepiride respectively. The most common admitting outcomes assessed were glycemia control, and the incidence of adverse drug reactions. When comparing the mean fasting and post prandial blood glucose obtained before starting the treatment with mean fasting and post prandial blood glucose levels recorded after zero month a statistically significant difference existed in both the groups.

These results are in support of a study mentioning that a significant decrease was noted in mean blood glucose levels in patients who were treated with combination of sitagliptin+metformin and glimepiride alone. ${ }^{15-17}$ When comparing the glycemia control achieved by combination therapy of sitagliptin+metformin a statistically significant difference was recorded among mean blood glucose levels for a period from zero month to six month. A significant difference $(\mathrm{P}<0.001)$ was noted in when HbA1c compared after starting the treatment with $\mathrm{HbAlc}$ recorded on Zero month treatment in group A who were treated with sitaglitin+metformin. These results are in accordance to a study mentioning the significant decrease in $\mathrm{HbA} 1 \mathrm{c}$ values in patients treated with combination of sitaglitin+metformin. ${ }^{18-21}$

In addition a significant difference also existed in HbAlc values recorded after the three and six months period when compared to HbA1c value recorded on zero month period in group- B who were treated with glimepiride alone. These results are in accordance to a study mentioning the significant decrease in $\mathrm{HbA} 1 \mathrm{c}$ values in patients treated with glimepiride alone. ${ }^{17}$ While comparing the incidence adverse drug reactions less number of hypoglycaemic episodes were recorded in patients who were treated with sitagliptin+metformin combination.

Whereas as compare to sitagliptin+metformin combination hypoglycaemic episodes were more in patient who were treated with glimepiride alone and this difference in hypoglycaemic episodes was statistically significant. These results are in accordance to previous studies. $^{22,23}$ Whereas the incidence of adverse drug reactions like GIT complications like nausea, vomiting and abdominal discomfort were recorded in both groups but this difference was statistically insignificant. Weight loss was recorded in patients who were treated with sitagliptin+metformin. These results are similar to previous studies showed significant reduction in weight. ${ }^{24}$ Whereas Weight loss was not observed but weight gain was recorded in patients who were treated with glimepiride alone. These results are in accordance to previous studies mentioned significant weight gain treated with glimeride. $^{22}$
The results of this study reveal that the combination regimen was more efficacious to achieve glycaemia control in comparison to monotherapy group. Therefore, sitagliptin+metformin as combination therapy for control of hyperglyacemia in type 2 diabetics result superior to glimepiride therapy. Time and resource constraint were the major limitation of this study therefore more short and long term studies are warranted to investigate the significance and causal relationships of the differences in the outcomes with the treatments.

\section{CONCLUSION}

The present study suggests that a significant difference may be existing in the clinical outcome interms of glycemia control and incidence of adverse drug reactions between combination of sitagliptin+metformin and glimepiride alone in type-2 diabetic patients. The combination of sitagliptin+metformin resulted comparatively in better glycemia control and less incidences of adverse drug reactions.

\section{Funding: No funding sources}

Conflict of interest: None declared

Ethical approval: The study was approved by the Institutional Ethics Committee

\section{REFERENCES}

1. Kumar PJ, Clark M. Diabetes mellitus and other disorders of metabolism. Textbook of Clinical Medicine. Pub: Saunders (London); 2002:1099-1121.

2. Expert Committee on the Diagnosis and Classification of Diabetes Mellitus. Report of the expert committee on the diagnosis and classification of Diabetes Mellitus. Diabetes Care. 1997;20:1183-97.

3. Beverley $B$, Eschwège $E$. The diagnosis and classification of diabetes and impaired glucose tolerance. In: Textbook of Diabetes 1 Ed: John C Pickup and Gareth Williams $3^{\text {rd }}$ ed; Chapter 2 2003;2.1-2.11.

4. Lindberg G, Lindblad U, Melander A. Sulfonylureas for treating type 2 diabetes mellitus. Cochrane Database Systemic Reviews. 2004;(3).

5. Amos A, McCarty D, Zimmet P. The rising global burden of diabetes and its complications, estimates and projections to the year 2010. Diabetic Med. 1997; 14:1-85.

6. King H, Aubert R, Herman W. Global burden of diabetes, 1995-2025. Prevalence, numerical estimates and projections. Diabetes Care. 1998;21:1414-31.

7. Zimmet P. Globalization, coca-colonization and the chronic disease epidemic: can the Doomsday scenario be averted. J Med. 2000;247:301-10.

8. Wild S, Roglic G, Green A, Sicree R, King H. Global prevalence of diabetes: Estimates for the year 2000 and projections for 2030. Diabetes Care. 2004;27:1047-53. 
9. UK prospective Diabetes study (UKPDS) Global prevalence of Diabetes: Estimate for the year 2000 and projections for 2030. Diabetes care. 2004;27:1047-53.

10. Turner RC, Cull, Fright V, Holman RR. Glycemic control with diet, Sulfonylurea, metformin, or insulin in patients with type 2 diabetes mellitus: progressive requirement for multiple therapies (UKPDS 49). UK prospective Diabetes study (UKPDS) Group. JAMA. 1999:281;2005-12.

11. Choy M, Lam S. Sitagliptin: a nobel drug for the treatment of type 2 diabetes. Cardiol Rev. 2007;15:264-71.

12. Inzucchi SE, Maggs DG, Spollett GR, Page SL, Rife FS, Walton V, et al. Efficacy and metabolic effects of metformin and troglitazone in type II diabetes mellitus. New Eng J Med. 1998 Mar 26;338(13):86773.

13. Product Information. JANUVIA (sitagliptin). White house Station: Merck and Co., Inc. October 2006.

14. Davidson Ja, parent EB, Gross JL. Incretin mimetics and Dipeptidyl Peptidase- 4inhibitors; Innovative treatment therapies for type 2 diabetes. Arq Bras Endocrinol Metab. 2008;52/6:1039-49.

15. Goldstein BJ, Feinglos MN, Lunceford JK, Johnson J, Williams-Herman DE. Effect of initial combination therapy with sitagliptin, a dipeptidyl peptidase-4 inhibitor, and metformin on glycemic control in patients with type 2 diabetes. Diabetes Care. 2007;30:1979-87.

16. Hermansen K, Kipnes M, Luo E, Fanurik D, Khatami H, Stein P. Efficacy and safety of the dipeptidyl peptidase-4 inhibitor, sitagliptin, in patients with type 2 diabetes mellitus inadequately controlled on glimepiride alone or on glimepiride and metformin. Diabetes Obes Metab. 2007;9:733-45.

17. Terauchi Y, Yamada Y, Ishida H, Ohsugi M, Kitaoka M, Satoh J, et al. Efficacy and safety of sitagliptin as compared with glimepiride in $\mathrm{J}$ apanese patients with type 2 diabetes mellitus aged $\geq 60$ years (START-J trial). Diabetes, Obesity and Metabolism. 2017 Aug;19(8):1188-92.

18. Raz I, Hanefeld M, Xu L, Caria C, Williams-Herman D, Khatami H. Efficacy and safety of the dipeptidyl peptidase-4 inhibitor sitagliptin as monotherapy in patients with type 2 diabetes mellitus. Diabetologia. 2006;49:2564-71.

19. Bennett WL, Maruthur NM, Singh S, Segal JB, Wilson LM, Chatterjee R, et al. Comparative effectiveness and safety of medications for type 2 diabetes: an update including new drugs and 2-drug combinations. Ann Intern Med. 2011;154:602-13.

20. Reasner C, Olansky L, Seck TL, Williams-Herman DE, Chen M, Terranella L, et al. The effect of initial therapy with fixeddose combination of sitagliptin and metformin compared with metformin monotherapy in patients with type 2 diabetes mellitus Diabetes Obes Metab. 2011;13:644-52.

21. Wainstein J, Katz L, Engel SS, Xu L, Golm GT, Hussain S, et al. Initial therapy with the fixed-dose combination of sitagliptin and metformin results in greater improvement in glycaemia control compared with pioglitazone monotherapy in patients with type 2diabetes. Diabetes Obes Metab. 2012;14:409-18.

22. Nauck M, Meininger G, Sheng DO, Terranella L, Stein PP. Sitagliptin Study 024 Group. Efficacy and safety of the dipeptidyl peptidase-4 inhibitor, sitagliptin, compared with the sulfonylurea, glipizide, in patients with type 2 diabetes inadequately controlled on metformin alone: a randomized, doubleblind, non-inferiority trial. Diabetes, Obes Metab. 2007 Mar;9(2):194-205.

23. Amori RE, Lau J, Pittas AG. Efficacy and safety of incretin therapy in type 2 diabetes: systematic review and meta-analysis. JAMA. 2007 Jul 11;298(2):194206.

24. Arechavaleta R, Seck T, Chen Y. Efficacy and safety of treatment with sitagliptin orglimepiride in patients with type 2 diabetes inadequately controlled on metformin monotherapy: arandomized, double-blind, non-inferiority trial. Diabetes Obes Metab. 2011;13(2):160-8.

Cite this article as: Tahashildar J, Singh RS,

Tahashildar J. Comparison of clinical outcomes of sitagliptin+metformin combination and glimepiride in the management of uncomplicated type 2

diabetics. Int J Basic Clin Pharmacol 2018;8:16-20. 